\title{
La inserción de las tic en el proceso de enseñanza y aprendizaje del contexto universitario ecuatoriano
}

\section{The insertion of ict in the teaching and learning process in the Ecuadorian university context}

DOI: $10.46932 / \mathrm{sfjdv2n2-179}$

Received in: March 1st, 2021

Accepted in: May 30th, 2021

\author{
Zoyla Leonor Toala Bazurto \\ Ingeniera Comercial \\ Docente en Nivelación de Carrera \\ Universidad Técnica de Manabí \\ E-mail: zoletoba1975@gmail.com
}

\section{Gustavo Adolfo Santana Sardi}

Licenciado en Ciencias de la Educación Técnica mención Contabilidad Computarizada

Diplomado en Desarrollo de Estrategias Digitales de Aprendizaje

Magister en Gerencia Educativa

Analista del Centro de Promoción y Apoyo al Ingreso

Universidad Técnica de Manabí

E-mail: gusansar@hotmail.com,gusansar@gmail.com,gustavo.santana@utm.edu.ec

\author{
Kerly Leomar Toala Vera \\ Licenciada en Ciencias de la Educación especialización Historia y Geografía \\ Magister en Docencia e Investigación Educativa \\ Analista del Centro de Promoción y Apoyo al Ingreso \\ Universidad Técnica de Manabí \\ E-mail: kerly.toala@utm.edu.ec; kerlyta13@hotmail.es
}

\section{RESUMEN}

A pesar de que la tecnología ha evolucionado con el pasar del tiempo y, desde que la informática comenzó a introducirse en la educación, muchos docentes no sólo no conocen mucho acerca de las nuevas tecnologías, sino que tampoco saben cómo manejar un computador o navegar en internet con un grado de conocimiento básico. Esto realmente se aprende mientras, otros docentes se capacitan para tener conocimiento aceptable en este campo y dan sus primeros pasos, y otros son además capaces de adquirir distintas formas y emplear las herramientas de información de modo creativo y de acuerdo a sus necesidades y las de sus alumnos. La incorporación de las tecnologías en la educación es un llamado que hace la sociedad y surge de la necesidad cada vez mayor del uso de la información. Se establecen así algunas características resaltantes de las TIC que permiten seleccionarlas como medio de instrucción y hasta en ocasiones como un ambiente ideal para el desarrollo del acto educativo, dependiendo del tipo de tecnología que se utilice. En este documento se establece una serie de puntos en las que se recogen las ventajas y posibilidades que las TIC aportan con su inserción a la formación favoreciendo el aprendizaje independiente, nuevas posibilidades para la orientación y facilitar la formación permanente en el proceso de enseñanza y aprendizaje del contexto universitario ecuatoriano. La técnica utilizada se encuentra enmarcado en una investigación documental, basada en una metodología descriptiva donde el criterio de varios autores se lo utiliza para realizar el contexto de este artículo. 
Palabras claves: Inserción, tecnologías de información y comunicación, proceso, enseñanza, aprendizaje, universitario ecuatoriano.

\begin{abstract}
Despite the fact that technology has evolved over time and, since computing began to enter education, many teachers not only do not know much about new technologies, but they also do not know how to operate a computer or navigate in internet with a degree of basic knowledge. This is really learned while, other teachers are trained to have acceptable knowledge in this field and take their first steps, and others are also able to acquire different forms and use information tools creatively and according to their needs and those of your students. Incorporation of technologies in education is a call made by the society and arises out of necessity every time greater use of information. Settle down thus some outstanding characteristics of the ICT that allow them to be selected as a medium of instruction and even sometimes like an ideal environment for the development of the act educational, depending on the type of technology that is used. This document establishes a series of points in which the advantages and possibilities that ICTs contribute with their insertion into training are established, favoring independent learning, new possibilities for orientation and facilitating permanent training, etc. in the teaching process and learning from the Ecuadorian university context. The technique used is framed in documentary film research, based on a descriptive methodology where the criteria of several authors is used to create the context of this article.
\end{abstract}

Keywords: Insertion, information and communication technologies, process, teaching, learning, Ecuadorian university.

\title{
1 INTRODUCCION
}

Las tecnologías han avanzado en forma vertiginosa en todos los ámbitos de la sociedad, incorporándose en forma progresiva en las Universidades, hasta convertirse en un recurso educativo muy valioso. En este contexto de cambios permanentes, las Instituciones de Educación Superior están llamada a hacer frente a los retos que suponen los nuevos métodos de aprendizaje, para mejorar la forma de producir, organizar y difundir el saber, dado que la integración de las TIC a la enseñanza encierran un gran potencial de aumento del acceso, la calidad y los buenos resultados. Desde mediados de los años 80 se empezó a hablar de tecnologías aplicadas a la información, adoptando diferentes definiciones durante décadas, siendo en el siglo XXI cuando se empieza a reconocer por algunos autores e instituciones el papel fundamental de las TIC en la sociedad (Vila et al., 2016) p. 249. Existe un gran apoyo de los gobiernos hacia las TIC, impulsado por la creación en 2009 del de Ministerio de Tecnologías de la Información y las Comunicaciones (MINTIC), antiguo Ministerio de Comunicaciones, para la promoción del acceso y uso de las TIC, así como para el desarrollo de las iniciativas necesarias para dar un salto en cobertura, expansión y superación de las barreras en pos de las proyecciones trazadas desde la directriz estatal (Duarte et al., 2016) p. 14. En su labor, el MINTIC ha reconocido la importancia de llevar las TIC a las aulas, impulsando iniciativas como la dotación de las aulas de clase de las Instituciones de Educación Superior oficiales con ciertos recursos tecnológicos, como estrategia de integración de toda la población, 
esto ha logrado explorar nuevos métodos de enseñanza y alternativas de capacitación que se extienden también al cuerpo docente (Cifuentes, 2016) p. 24.

Para llevar a cabo el proceso de enseñanza y aprendizaje, el docente cuenta con diversidad de herramientas que le permite interactuar con los alumnos para fomentar su participación, motivación e interés por el tema tratado, con la finalidad de transmitir el conocimiento que posee de una manera significativa (Torres y Velandia, 2017) p. 125.

Organismos como la ONU, CMSI, UNESCO entre otros han propuesto objetivos y líneas de acción muy claras para la inclusión de las TIC en la educación y proyectos educativos, con la idea de que los gobiernos de turno fomenten su inserción en cada país, aunque de esto mismo no se conoce cuál es el grado de apoyo de cada país para este proceso. Sin embargo, el proceso de inserción de las TIC en las aulas corresponde a un arduo trabajo. La simple incorporación de dispositivos electrónicos en el aula no significa que la educación está siendo transformada. Existe una serie de fases por las que se debe pasar para poder reorganizar los sistemas en las Instituciones de Educación Superior de manera que estos se adapten a modelos de formación centrados en el trabajo y aprendizaje del estudiante, todo esto apoyado por las TIC. Una de las actividades realizadas por el expresidente de la República Rafael Correa se ha presenciado en Ecuador grandes esfuerzos por incluir en la política nacional el uso de las TIC en la sociedad en general y en las Instituciones de Educación Superior (INEC, 2017) p. 12.

La investigación ha tenido bastante atención por parte de varios autores y organismos a nivel mundial. En las últimas décadas, se ha generado una variada producción científica sobre el tema de las TIC en el ámbito de la educación. La importancia que se le ha dado a la inserción de las TIC en las Instituciones de Educación Superior y la forma en que estos gobiernos han hecho frente la inserción a través de políticas públicas y proyectos implantados. La organización educativa en Ecuador se delega las competencias educativas al respectivo Ministerio de Educación del país es decir sigue un modelo nacional-regional.

Este artículo se desarrollará mediante un análisis descriptivo analítico que permita caracterizar la inserción de las TIC en el proceso de enseñanza y aprendizaje del contexto universitario ecuatoriano, Para esto es necesario analizar publicaciones científicas. También se realizará un trabajo analítico, puesto que se estudiarán las variables que influyen en la inserción de las TIC.

\section{DESARROLLO}

Es necesario partir de la idea de que las TIC han presentado en las últimas décadas una incursión significativa en casi todos los aspectos de la humanidad; y la educación ha sido uno de ellos. Las TIC en las Instituciones de educación Superior representan una herramienta que desde diferentes puntos de vista 
ha motivado reflexiones positivas y negativas acerca de su contribución en los procesos de enseñanza y de aprendizaje, y son contempladas en los requerimientos educativos internacionales, nacionales y locales de cualquier país.

La investigación en torno a las TIC ha tenido también un crecimiento revelador y el interés de estas ha estado enfocado especialmente en la presentación de resultados obtenidos a partir de la implementación de estas herramientas en el aula y el mayor uso de algunas de estas en la práctica docente, es evidente como en los últimos años la integración de las TIC en las Instituciones de Educación Superior se ha convertido en centro de atención en el ámbito educativo pues de esto se pueden encontrar numerosas publicaciones, eventos científicos, investigaciones, experiencias, proyectos, entre otras, surgidos sobre el tema. Además de aplicar las nuevas tecnologías a la educación hay que diseñar nuevos escenarios educativos donde los estudiantes puedan aprender a utilizar e intervenir en el nuevo sistema educativo. Sin embargo para que todo esto llegue a ser posible debe pasar por un sinnúmero de fases hasta alcanzar el fin que se desea y superar varios obstáculos que esta inserción representa. En esto hay que hacer hincapié pues tal como lo recalca Julio Cabero las tecnologías, independientemente de lo potente que sean son independientemente de lo potente que sea son solamente instrumentos curriculares, y por tanto su sentido, vida y efecto pedagógico, vendrá de las relaciones que sepamos establecer con el resto de componentes del currículum. Es decir el resultado final o efecto que se consiga de la inserción de la tecnología en las Instituciones de Educación Superior será de la interacción que resulte de esta con todos los elementos pedagógicos ya existentes y las metodologías que aquí se apliquen. Pues como lo explica J.Cabero (2018) p. 4 "El poder no está en la tecnología, sino en las preguntas y respuestas que nos hagamos sobre ella para su diseño, y utilización en investigación en la enseñanza”. La enseñanza tradicional no los puede preparar de una forma óptima porque el uso de esas tecnologías es uno de los nexos que los unen y los definen como generación o colectivo.

Para el autor (Velázquez, 2017), citado por V.Vélez, M. E., Zambrano, N. L., Intriago Cedeño, M. E., Santana Sardi, G. A., y Toala Vera, K. L. (2020) p. 982, las TIC son el conjunto de metodologías desarrolladas para tramitar información y así poder remitirla de un lugar a otro. Esta definición nos encamina a la principal acción concebida para los usuarios de las TIC: gestionar. En cuanto a herramientas tecnológicas varios docentes usan las TIC como apoyo en el proceso de enseñanza y aprendizaje, implementando varias estrategias de aprendizaje en el aula de clase, para de esta manera propiciar escenarios educativos dinámicos y motivadores que faciliten el desarrollo del aprendizaje autónomo de los estudiantes. (Aedo, Ramos, \& Romero, 2018), un ambiente de aprendizaje que utilice las tecnologías entrega variadas oportunidades y beneficios a los estudiantes y profesores, además de cambios en los roles. Las TIC juegan un papel importante en la educación superior, son necesarios para revolucionar el 
conocimiento, el docente debe hacer uso de todos ellos para que el estudiante llegue a comprender su clase de manera dinámica, la transmisión del conocimiento a través del uso de las tecnologías a proporcionado más conocimiento, ya que este se encuentra disperso en la nube, y ayuda a solucionar problemas cuando algo no se entiende.

Según Portilla (2017), el empleo de las TIC en las prácticas educativas se mantiene con un uso limitado, que se mantiene por debajo de su potencial. Esta situación evidencia la relevancia de las concepciones y pensamiento educativo como fundamento e impulso de toda innovación. Desde mediados de la década de 2000 se comienza a trabajar en la incorporación de las TIC en el proceso de enseñanza y de gestión institucional. Comienza un proceso de virtualización de la enseñanza y se expresa en ofertas bimodales; aumento de los apoyos de recursos didácticos digitales al proceso de enseñanza; incorporación de asignaturas asociadas a la formación de competencias informáticas e informacionales; automatización de los procesos académicos; oferta de cursos virtuales; y aprendizajes en sistemas de simulación e interacción docente-estudiante a través de campus virtuales (Farfán, 2016).

Un estudio realizado por Benítez, Hernández y Pichs (2016) revela que las principales tendencias en este sentido están impulsadas por los retos que enfrentan los sistemas y las instituciones, pudiéndose identificar entre los más importantes cambios a que el proceso docente debe estar centrado en el aprendizaje con la utilización de medios modernos de enseñanza para lograr habilidades, conocimientos y valores en los egresados que respondan a la calidad que se exige. (Cano, 2019). Para potenciar la introducción de las TIC a los procesos formativos en pos de la educación virtual, se ha diseñado un modelo para la virtualización de la actividad formativa, lo cual contribuye a la apertura de nuevas formas de enseñar y aprender, en correspondencia a las tendencias actuales de la educación.

Como lo menciona R. Báez (2007) p. 309, los procesos de enseñanza y aprendizaje involucran el currículo, el alumno, el docente y las estrategias metodológicas. En el caso de la enseñanza y el aprendizaje, las TIC contribuye a que ésta sea administrada con una visión integradora, holística e investigativa, a fin de que el educando obtenga un aprendizaje significativo. Para ello, es necesario cumplir con los principios que son fundamentales para abordar el proceso de enseñanza y aprendizaje. Dichos principios se describen a continuación:

- Principio globalizador e interdisciplinar: Este principio consiste en una aproximación consciente a una realidad compleja, en la que cada uno de los elementos que la forman está estrechamente interrelacionado con los demás y unos son consecuencias de otros.

- Integración del conocimiento: Se parte del principio que el conocimiento no puede ser fragmentado, el mismo debe ser holístico, integrador y complejo, donde el saber es como un todo, 
integrado. Para ello se deben integrar los objetivos, los contenidos, la metodología y la evaluación, así como la relación entre sí. Conjugando al mismo tiempo la teoría con la práctica.

o Contextualización del conocimiento: Implica adaptar los contenidos a las características y necesidades de los alumnos a quienes se dirige la enseñanza, partiendo de situaciones reales y cotidianas conocidas por el alumno, en otras palabras, de hechos concretos.

- Principio de flexibilidad: Todo proceso de enseñanza parte de una planificación, la misma debe responder a las necesidades e intereses de aprendizaje de los alumnos, por tanto, su organización y administración debe ser flexible, adaptable a los requerimiento del educando, sin perder de vista lo que se quiere trabajar, que orden y secuencia deben presentarse los contenidos.

○ Aprendizaje por descubrimiento: Implica considerar la participación del alumno dentro de su formación, dejando de ser un mero receptor pasivo para convertirse en elemento activo y motor de su propio aprendizaje, propiciando en él, la investigación, la reflexión y la búsqueda o indagación del conocimiento.

- Innovación de estrategias metodológicas: Consiste en hacer uso de estrategias que inviten al descubrimiento, la investigación y la construcción del aprendizaje; estas estrategias pueden ser individuales y grupales, las mismas se pueden implementar en función a: la organización de los contenidos, la exposición de los contenidos y las actividades del alumno.

Como lo menciona (G. Santana-Sardi, Francisco Antonio Mawyin-Cevallos, Jhimmy Andrés Gutiérrez-Santana, Laura Leonor Santos-Moreira, Geilert De la Peña) (2020) p. 337, estas transformaciones han de expresarse fundamentalmente en la renovación de concepciones y prácticas pedagógicas que implican reformular el papel del docente y desarrollar modelos de aprendizaje distintos a los tradicionales, en los que el aprendizaje y desarrollo de las novedades en el empleo de las TIC es de vital importancia en el quehacer docente, impulsado por las exigencias actuales y las realidades que se viven en las condiciones de la docencia de las Instituciones de Educación Superior en el Ecuador en estos días.

Las Instituciones de Educación Superior en general, ha propiciado introducir las tecnologías de la información y las comunicaciones como un elemento innovador en el proceso de enseñanza aprendizaje y además, estimula generar nuevas funciones que constituyan un valor añadido de los recursos que los docentes tengan a su disposición, y que hoy se evidencia su mayor impacto, necesidad e importancia para sostener todo el sistema educativo.

La integración de las Tecnologías de la Información y la Comunicación (TIC) en los procesos de enseñanza-aprendizaje, conlleva numerosos cambios. Por una parte, cabe señalar las modificaciones que se producen a nivel de infraestructuras tecnológicas, por otro lado, los cambios que se producen a nivel 
del docente y de los alumnos (Vera, Torres \& Martínez, 2014). El rol del docente (De Juanas \& Fernández, 2008; Abad, García, Magro \& Serrano, 2010) pasa de centrarse en transmitir los contenidos, a estimular la búsqueda personal del conocimiento por parte del alumno.

Berenguer, (2016) p. 1468, por su parte plantea: “(...) este modelo educativo sería la de promover que el alumno trabaje por sí mismo y fuera del aula los conceptos teóricos a través de diversas herramientas que el docente pone a su alcance, principalmente vídeos o podcasts grabados por su profesor o por otras personas (pero no exclusivamente), y el tiempo de clase se aproveche para resolver dudas relacionadas con el material proporcionado, realizar prácticas y abrir foros de discusión sobre cuestiones controvertidas." En el contexto que se analiza resulta muy útil en estos momentos, aunque se han determinado que significativamente muchos docentes y estudiante son poseen todas la competencias y habilidades para su uso óptimo en sus procesos. Sin embargo, para docentes y estudiantes este ofrece un servicio automatizado y personalizado a sus necesidades e intereses, lo que favorece el ritmo individual y las diferentes perspectivas o desempeños de aprendizaje. El acceso al conocimiento de la asignatura a través de secuencias didácticas de aprendizaje facilita de una manera sencilla e intuitiva la navegación por el curso.

En el caso de las sesiones de trabajo directo por herramientas de video conferencia como : Zoom, Skype, Google Hangouts, Google Meeting, Google Classroom, como las más utilizadas, y en correspondencia en la normalidad la modalidad semipresencial, la plataforma se utiliza como medio para colocar contenidos y actividades sobre los que se trabajará posteriormente en las sesiones presenciales, o también en las sesiones de trabajo por las video conferencias o interacción sincrónica en las actividades de aprendizaje. (Larraguivel, 2020).

El Ministerio de Educación del Ecuador MINEDUC, hizo lineamientos que orientaban cómo se deberían dar las clases virtuales y sobre todo se buscó enfatizar en la importancia de la planificación curricular, la cual asegura que el proceso de aprendizaje sea continuo, pertinente y en especial que sea dosificado. Todo este procedimiento permite que los docentes puedan organizar y dirigir de una manera adecuada cada una de las necesidades educativas que tienen los estudiantes. En una época de emergencia la planificación es necesaria y gracias a ella los docentes pueden generar rutinas y actividades coherentes, claras y mejorables de esa forma los beneficiados son los estudiantes. Es necesario que el docente busque generar en sus estudiantes dudas sobre cómo tener un autocuidado en esta pandemia, (Ministerio de Educación del Ecuador, 2020, p.10).

Al hablar de las nuevas tecnologías en el proceso de enseñanza y aprendizaje del contexto universitario ecuatoriano, es necesario hacer referencia a la relación que ha de establecerse en el uso de nuevos medios e innovación educativa universitaria. Es interesante la propuesta de De Pablos, Colás y 
González (2010), al establecer tres niveles en relación con la implantación de las tic en el sistema educativo: introducción, aplicación e integración. Si pretendemos alcanzar un nivel de integración, identificado con la plena incorporación de las tic al sistema educativo, debemos superar los dos niveles anteriores.

- La etapa de introducción implica la correspondiente dotación de los medios a los centros educativos y su familiarización por parte de los docentes y estudiantes.

- La etapa de aplicación se encuentra en situaciones en las que, superado un conocimiento o dominio instrumental, se van descubriendo las aplicaciones pedagógicas básicas de estos medios en cada campo específico de la actividad docente.

Así pues, las distintas investigaciones indican que la implantación de las tic en las Instituciones de Educación Superior se sitúa fundamentalmente en los primeros niveles de introducción y aplicación (De Pablos, Colás y González, 2010).

Las TIC se ensalzan en cuanto uno de los pilares fundamentales en la construcción del conocimiento, llegando a configurar la formación actual en el mundo universitario, compaginando presencialidad y virtualidad en todas sus formas, además de las virtuales. De tal suerte, las tic ofrecen nuevas posibilidades como la de formar a un sector de la población que tenía dificultades en el acceso regular a las aulas, la continuidad de formación permanente adaptándose con mayor eficacia a las necesidades personales y laborales de los estudiantes o las multiplicidad de modos de comunicación, vivencia, valoración y transformación social que brindan (García-Valcárcel et al., 2015).

En relación con los beneficios que proporciona la incorporación de las tic en las Instituciones de Educación Superior, encontramos los siguientes: facilidad de acceso y gestión de la información; transversalidad que incorporar a los procesos de gestión, formación e investigación; mejora en la organización de la innovación, al mejorar la estructura lógica; comprensión de contenidos a partir de materiales multimedia; estímulo a la independencia del estudio, disminuyendo la dependencia a las procesos de enseñanza-aprendizaje tradicionales; ampliación de la comunicación entre docentes y alumnado y apertura a otros miembros de la comunidad científica, así como desarrollo de competencias vinculadas con tic en el profesorado y alumnado universitario (García-Valcárcel et al., 2015 ; Bosco, 2005; Duart y Lupiáñez, 2005).

La transformación tecnológica constante y la innovación tienen efectos a nivel productivo, económico, social y cultural, pero también educativo. En el último Informe Horizon para la Institución de Educación Superior, Johnson, Adams y Cummins (2012) establecen las tendencias, derivadas de la evolución tecnológica, para los próximos cinco años. En ellas se vislumbra, a corto plazo, el uso de tabletas y dispositivos móviles con contenidos educativos; a mediano plazo, el aprendizaje 
basado en juegos y las analíticas de aprendizaje con una mayor presencia en las enseñanza a nivel universitario y a más largo plazo de acuerdo con el desarrollo e implantación que tenga el aprendizaje basado en gestos y el Internet acabarán penetrando por completo la enseñanza de Educación Superior. La incorporación de las TIC en las Instituciones de Educación Superior ha provocado cambios en la manera de entender y señalan que el papel que pueden tener las TIC en el aprendizaje personalizado, en la tutoría y seguimiento individualizado de los estudiantes; y en la posibilidad de un aprendizaje social por medio de las redes y de herramientas de trabajo colaborativo.

Es importante insistir que el éxito de la educación medida por las TIC depende en gran medida de la comprensión, preparación y capacidad del docente para trasformar las ortodoxas maneras de enseñar y aprender, a través de poner al servicio del proceso de enseñanza-aprendizaje las potencialidades de las herramientas y recursos de estas tecnologías. Estas tecnologías son poderosos medios para la implementación de modelos de innovación educativa en ambientes de aprendizaje constructivistas apoyados en novedosos procedimientos y métodos de enseñanza y aprendizaje orientados al desarrollo significativo del conocimiento humano (Díaz-Vidal, 2013; UNESCO, 2018).

Ya se ha analizado la necesidad de contar con recursos humanos con conocimientos sobre las tecnologías disponibles para la implementación de las TIC en los ambientes áulicos, pero en este análisis no puede faltar otro elemento fundamental que, es el conocimiento de las metodologías adecuadas para hacer uso de las potencialidades de estas tecnologías. Existen múltiples autores que abordan esta temática, entre los que se encuentran Aguilera-Ruiz (2017), Gaviria-Rodríguez (2019) y Godoy (2019) p. 25, quienes proponen las metodologías activas como vía para garantizar el aprendizaje significativo de los estudiantes en los diferentes niveles de enseñanza; entre estas metodologías apoyadas por las TIC significan la gamificación, el aula invertida y enseñanza a distancia (e-learning). La gamificación consiste en la utilización de videos-juegos con propósitos educativos se pretende que el estudiante bajo el pretexto de jugar aprenda de manera significativa; esta novedosa metodología despierta el interés de los estudiantes condicionando sus estructura cognitivas para la apropiación de los nuevos conocimientos; además, desarrolla en ellos competencias para el aprendizaje autónomo, actitudes cooperativas y empatía entre los pares, todo lo que repercute en un mejor rendimiento académico(Ortiz, Jordán y Agreda, 2018) p. 12.

En esencia esta metodología consiste en la realización de actividades didácticas previas como punto de partida para posteriormente desarrollar el proceso de forma presencial sobre los resultados obtenidos. Esta preparación del estudiante fuera de los salones de clase está apoyada en la gestión del docente como facilitador mediante guías, presentaciones digitales, medios 
audiovisuales y otros materiales que, orientan el trabajo del discente en la búsqueda de información a través del empleo de las TIC (Aguilera-Ruiz et al., 2017) p. 262.

Las averiguaciones realizadas apuntan a que aún son insuficientes las acciones que se realizan en este sentido; las investigaciones llevadas a cabo por Suasnabas -Pacheco et al. (2017), Espinoza, et al. (2018) y Palacios-Valderrama, et al. (2018) p. 734, dan fe de esta situación; la que se caracteriza por insuficiente formación tecnológica con fines educativos de los docentes que, trae por consecuencia el poco uso y variedad de las TIC como materiales de apoyo a la docencia, desidia de algunos docentes por el uso de las tecnologías digitales con fines educativos limitando éstas a funciones instrumentales; el mayor uso dado a estas tecnologías es en la planificación de las clases con el apoyo de los procesadores de texto y para los trabajos administrativos con los tabuladores electrónicos. Otro importante factor que frena la implementación de las TIC en las aulas ecuatorianas es la persistencia de las metodologías de enseñanza y aprendizaje tradicionales que, son utilizadas desde hace mucho tiempo y deben ser superadas. Asimismo, en las instituciones educativas, en muchos casos, no existe un proyecto para la implementación de estas tecnologías, por lo que las disposiciones para su implementación se convierten en letra muerta.

\section{CONCLUSIONES}

La inserción de las TIC en el contexto universitario ecuatoriano se evidencia en la infraestructura y recursos tanto en el nivel de administración y servicios como en los procesos de formación e investigación. Su integración en la educación superior se sustenta, por un lado, en la respuesta a la indudable necesidad que surge en la adaptación a los constantes cambios tecnológicos, y, por otro lado, en los beneficios que conlleva su incorporación en los órdenes organizativo, metodológico, formativo, motivador y comunicativo.

La investigación como parte analítica y descriptiva, aportó con elementos de análisis que se detallan la inserción de las TIC como objeto de estudio en los procesos de enseñanza y aprendizaje, teniendo en cuenta que éstos son procesos sociales, comunicativos y contextualizados en el marco de un currículo en el contexto Universitario Ecuatoriano.

Ante un mundo en constante proceso de cambio, la educación sigue siendo la respuesta pedagógica estratégica para dotar a los estudiantes de herramientas intelectuales, que les permitirán adaptarse a las incesantes transformaciones del mundo laboral y a la expansión del conocimiento. Por ello, la necesidad de la planificación y el uso de estrategias docentes que potencien aprendizajes reflexivos y una educación para afrontar los cambios, la incertidumbre y la dinámica del mundo actual, se fundamenta en la actualidad, entre otros aspectos por: el crecimiento vertiginoso de la información y la 
infinitud del conocimiento humano; el acelerado avance de las Tecnologías de la Información y las Comunicaciones; la proyección del aprendizaje a lo largo de toda la vida, lo cual pone al docente ante la necesidad de preparar a los estudiantes para que puedan aprender por sí mismos y sean capaces de dirigir su propio aprendizaje, a través del dominio consciente de sus recursos para generar estrategias y definir, emplear y evaluar los procedimientos necesarios para resolver problemas, atendiendo a las condiciones del medio y a las suyas propias; los nuevos modos de aprender, basados en el descubrimiento y la participación, con sistemas más flexibles, que permitan incorporar las herramientas tecnológicas para la búsqueda de información y compartir problemas, proyectos y tareas en la vida cotidiana.

Por otro lado, el Ecuador cuenta con un marco normativo de la actividad educativa basada en las TIC; sin embargo, en la práctica se observa falencias a saber: insuficiente formación tecnológica con fines educativos de los docentes, poco uso y variedad de las TIC como materiales didácticos, desidia de docentes por el uso de las tecnologías digitales, persistencia de las metodologías de enseñanza y aprendizaje tradicionales, por lo que la implementación de estas tecnologías en los procesos educativos de las diferentes enseñanzas aún es una tarea por cumplir.

Este artículo servirá también para realizar futuras investigaciones desde otras perspectivas. De hecho, la inserción de las TIC en el proceso de enseñanza y aprendizaje en el contexto universitario ecuatoriano, abre un abanico de posibilidades para todos los ámbitos. Así mismo, es importante evidenciar que el empleo efectivo de las diversas aplicaciones TIC mucho depende de los docentes y de la formación que hayan recibido. Por todo lo expuesto se subraya la necesidad de formar nuevos profesionales, con nuevas competencias y con mayor capacidad, para enfrentar con éxito los paradigmas tecnológicos en los contextos educativos actuales. 


\section{BIBLIOGRAFÍA}

Aedo, R. R., Ramos, E. C., \& Romero, M. E. (11 de Febrero de 2018). Un Modelo De Autoaprendizaje Con Integracion De Las Tic Y Los Metodos De Gestion Del Conocimiento. Obtenido de Un Modelo De Autoaprendizaje Con Integracion De Las Tic Y Los Metodos De Gestion Del Conocimiento: https://biblioteca.org.ar/libros/141704.pdf.

Aguilera-Ruiz, C., Manzano-León, A., Martínez-Moreno, I., Lozano-Segura, M. D., y Casiano Yanicelli, C. (2017). El Modelo Flipped Classroom. International Journal Of Developmental And Educational Psychology, 4(1), 261-266

Benítez, F., Hernández, D., y Pichs, B. (2016). Las transformaciones en la formación de profesionales. Panorama internacional. Revista Congreso Universidad, 5(1), pp. 171-186.

Berenguer-Albaladejo, C. (2016). Acerca de la utilidad del aula invertida o flipped classroom.

Cano, E. (2019). Evaluación por competencias en la Educación Superior: Buenas prácticas ante los actuales retos. RIEE. Revista Iberoamericana de Evaluación Educativa.

Cifuentes, G. A., (2016) Conceptualizando Prácticas de Liderazgo de las TIC: Un Estudio en la Educación Superior Colombiana, Education Policy Analysis Archives / Archivos Analíticos de Políticas Educativas, 24.

Colás, P., González, T. y Pablos, J. de (2013). Juventud y redes sociales: Motivaciones y usos preferentes. Comunicar, 40, 15-23.

De Juanas, A. \& Fernández, P. (2008). Competencias y estrategias de aprendizaje. Reflexiones sobre el proceso de cambio en el EEES. Cuadernos de trabajo social, 21, 217- 230.

Díaz-Vidal, J. (2013). La evaluación del aprendizaje y las TIC. Universidad Médica de Granma. Cuba

Duarte, M.A., A. A. Suárez y C. A. Suárez, (2016) Políticas y Programas del Sistema Educativo Colombiano como Marco para la Articulación de las TIC, Revista ALETHEIA, 8(1), 12-31.

Farfan, P. (2016). Modelo de virtualizacion educativa de la Universidad Politecnica Salesiana del Ecuador (Tesis Doctoral). Universidad Politecnica Salesiana, Cuenca, Ecuador.

GarcíaValcárcel, A., Basilotta, V., Cabezas, M., Casillas, S., González, L., Hernández, A. y Mena, J. J. (2015). La formación del profesorado universitario en Tecnologías de la Información y la Comunicación en la Universidad de Salamanca. RELATEC. Revista Latinoamericana de Tecnología Educativa, 14(1), $75-88$

G. Santana-Sardi, Francisco Antonio Mawyin-Cevallos, Jhimmy Andrés Gutiérrez-Santana, Laura Leonor Santos-Moreira, Geilert De la Peña (2020) p. 337. Buenas prácticas de enseñanza-aprendizaje con el empleo de clases invertidas para la formación continua en tiempos de Covid-19.

Godoy, M. (2019). La gamificación desde una reflexión teórica como recurso estratégico en la Educación. Revista Espacios,40 (15), p 25. 
INEC (2017). "Encuesta Nacional de Ingresos y gastos de los hogares", 2017. Disponible en http://www.inec.go.cr/encuestas/encuesta-nacional-de-ingresos-y-gastos-de-los-hogares, fecha de última consulta 19-03- 2018.

J Cabero (2018). "Mitos de la sociedad de la información: sus impactos en la educación". AGUIAR, M.V. y otros (coods): Cultura y Educación en la sociedad de la información, A Coruña, Netbiblo. 26-26.

Larraguivel, M. E. R. (2020). La práctica docente universitaria en ambientes de educación a distancia: tensiones y experiencias de cambio.

Ministerio de Educación del Ecuador. (2020, 12 de marzo). Comunicado Oficial Suspensión de las actividades académicas para los estudiantes para precautelar la salud de la comunidad educativa. Recuperado de https://educacion.gob.ec/comunicado-oficial-suspension-de-las actividades-académicaspara-los-estudiantes-para precautelar-la-salud-de-la-comunidad-educativa/.

Ortiz, C., A., Jordán, J. yAgredal, M. (2018). Gamificación En Educación: Una Panorámica sobre el estado de la cuestión. Revista Educação e Pesquisa, 44, 1-17.

Portilla, G. (2017). Concepcion teorico-metodologica para el empleo innovador de tecnologias educativas emergentes (TEE) en la asignatura Sociedad y Cultura de la Nivelacion de Carrera de la Universidad Nacional de Educacion (UNAE) (Tesis Doctoral), Azogues, Cañar, Ecuador.

R. Báez (2007) principios didácticos a seguir en el proceso de enseñanza y aprendizaje en la upel "El Mácaro" p. 309.

Suasnabas-Pacheco, L., Washington, F. Avila-Ortega, W., Díaz-Chong, E. y. Rodríguez-Quiñonez, V. (2017).Las Tics en los procesos de enseñanza y aprendizaje enla educación universitaria. Revista $\begin{array}{lllll}\text { Dominio de las. } & \text { Ciencias, } & \text { 721-749. }\end{array}$ http://dx.doi.org/10.23857/dom.cien.pocaip.2017.3.2.721-749

Torres, J.M.P. y S.R Velandia, (2017) Influencia de las Estrategias Pedagógicas en los Procesos de Aprendizaje de los Estudiantes de una Institución de Básica Primaria de la Ciudad de Bucaramanga, Puente, 7(2), 117-130.

UNESCO (2018). Las Tecnologías de la Información y la Comunicación (TIC) en la Educación.http://www.unesco.org/new/es/havana/areas-of-action/education/tic-en-la-educacion/.

Vélez Vélez, M. E., Zambrano Zambrano, N. L., Intriago Cedeño, M. E., Santana Sardi, G. A., \& Toala Vera, K. L. (2020). La comunicación educativa en el proceso de formación profesional por competencias. Dominio de las Ciencias, 6(4), 1357-1375.

Vila, R.R., S. Mengual-Andrés y C. L. A. Robles, (2016) ICT and New Educational Horizons in the Complex Society, In Education and Innovation in the University: Comparative Study between Italy and Spain, 239-251 